\title{
First Demonstration of a Modular and Reconfigurable Magnetic-Manipulation System
}

\author{
Andrew J. Petruska, Joseph B. Brink, and Jake J. Abbott
}

\begin{abstract}
This is the first demonstration of a modular and reconfigurable magnetic-manipulation system with integral ferromagnetic material. This system-which includes multiple Omnimagnets, each comprising three orthogonal solenoids and a spherical ferromagnetic core-is capable of dexterous manipulation of a magnetic tool. The magnetization coupling of an arbitrary arrangement of spherical ferromagnetic cores is solved, enabling an analytical solution for the magnetic forces and torques. Thus, the system does not require extensive field maps or in situ field characterization. If the positions and orientations of the Omnimagnets are sensed, the Omnimagnets can be actively rearranged during manipulation while maintaining control of the tool. This could enable new capabilities in medical procedures because the manipulation system can be modified to accommodate the imaging systems and enable physician access without loss of control. The capabilities of this system are demonstrated through five-degree-of-freedom manipulation (position and heading) of a permanent-magnetic tool with several arbitrary Omnimagnet configurations.
\end{abstract}

\section{INTRODUCTION}

There is significant interest in magnetic-manipulation systems for current and future minimally invasive medical applications [1]-[3]. Magnetic-manipulation systems apply forces and torques to remote magnetic devices without a mechanical connection, and can project this control unhindered through most environments, including biological tissue and plastics, as well as through nonferrous metals in a bandwidth-limited sense. Magnetic manipulation is also compatible with most medical-imaging modalities.

Many prior magnetic-manipulation systems have been designed so that the control of the field magnitude and direction (and the resulting applied torque) and field gradient (and the resulting applied force) can be separated and linearized. This is typically accomplished using combinations of Helmholtz and Maxwell coil pairs [4]-[7]. Clinical MRI systems are similarly designed, and can be modified for a similar type of manipulation [8], [9]. This separation and linearization requires precise design and placement of the coils to surround the workspace, leading to challenges in imaging and scalability.

If the generation of the field and the field gradient are not separated by design, which is the case for the most highperforming magnetic-manipulation systems demonstrated to date, then characterization of the magnetic field throughout

This work is supported by the National Science Foundation under Grant No. 0952718, and by the National Aeronautics and Space Administration under Grant No. NNX13AL46H.

The authors are with the Department of Mechanical Engineering, University of Utah, USA. A. J. Petruska is now with the Multi-Scale Robotics Laboratory, ETH Zurich, Switzerland.

andrewpe@ethz.ch, joseph.brink@utah.edu, jake.abbott@utah.edu

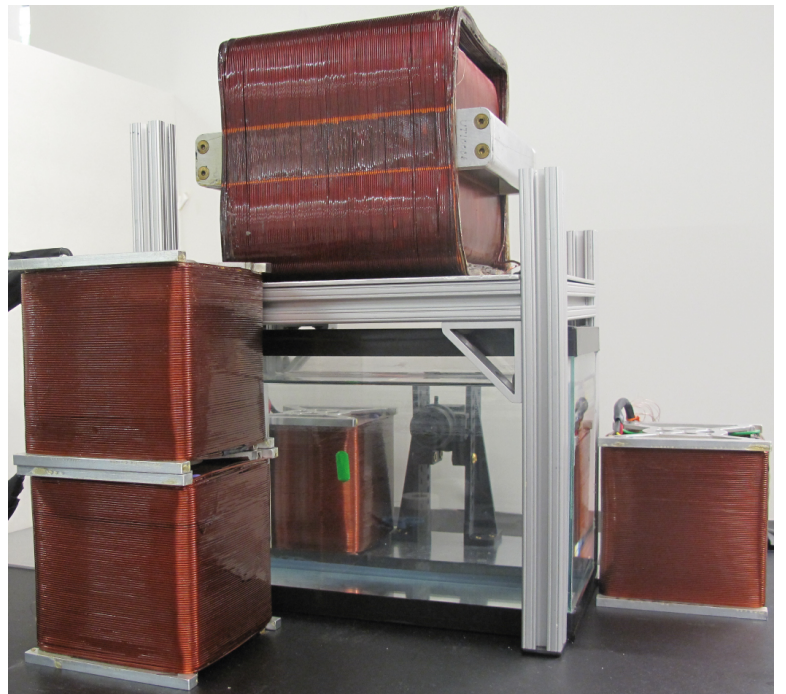

Fig. 1. Five-degree-of-freedom manipulation of a magnetic capsule in water, using five Omnimagnets. The Omnimagnets are arranged arbitrarily, and no in situ magnetic field characterization is required.

the workspace as a function of applied current is required. It is typical to use ferromagnetic materials with high coercivity and low remanence to enhance the field produced by the solenoids [10]-[14], although some systems have utilized air-core electromagnets using similar control methods [15]. In either case, the resulting field is a complex function of the shape, position, and orientation of each solenoid and all integral ferromagnetic materials, and no general analytical solution exists to determine the field resulting from an arbitrary arrangement of electromagnets. However, the magnetic field of a static arrangement of electromagnets is linear with the solenoid currents (up to the point of saturation of any ferromagnetic material), which leads to a method for realtime control with such systems: one field map per solenoid in situ is generated offline, and the individual field maps are then linearly combined online. If any component in the system is moved, the field must be completely remapped. It may be possible in certain cases to move system components in a constrained fashion such that the coupled field is minimally affected [13], but this is not the case in general.

We recently presented the design of the Omnimagnet [16], which is an omnidirectional electromagnetic cube comprising three densely packed mutually orthogonal solenoids surrounding a spherical ferromagnetic core. The Omnimagnet was optimized to be magnetically modeled by the analytical point-dipole model and is capable of three-degree-of- 
freedom (3-DOF) manipulation in an external workspace [17]. Originally, the Omnimagnet was conceived as an individual module for a reconfigurable magnetic-manipulation system (Fig. 1) capable of dexterous manipulation. In such a system, each additional Omnimagnet adds three additional inputs to the system, improving the system's manipulability and reducing the power required. The same set of Omnimagnets could be utilized in various arrangements that are optimized for a specific application, including optimizing for a specific medical procedure and location in the human body, optimizing within the constraints of a specific medicalimaging modality, or optimizing for a specific size of patient so there is no need to overdesign a one-size-fits-all system.

In our original conception of this modular and reconfigurable system, calibration and control would be handled analogously to prior magnetic-manipulation systems that contain integral ferromagnetic material, using in situ field maps. However, we have discovered that Omnimagnets' spherical cores make them particularly well suited for use in modular and reconfigurable systems. Spherical ferromagnetic objects have a property that is unique to their geometry: the average magnetic field applied over the volume of a sphere is always equal to the magnetic field applied at the center of the sphere. We will show that a consequence of this property is that the coupled magnetic field of an arbitrary number and arrangement of Omnimagnets can be accurately computed algebraically, rather than with the field-map method required previously. This means that a system of Omnimagnets not only represents the first truly modular and reconfigurable magnetic-manipulation system, but they are capable of being reconfigured in real-time, with Omnimagnets being moved, added, and removed from the system without any additional calibration step. Real-time reconfiguration capability will enable a physician to maintain magnetic control over the remote device while optimizing the location of the Omnimagnets for improved performance or improved access to the patient while accommodating the changing viewing perspectives of line-of-sight imaging systems (e.g., fluoroscopy).

In this paper, we describe the analytical method to characterize and control an arbitrary system of Omnimagnets, and we demonstrate dexterous manipulation of an untethered magnetic device using a multiple Omnimagnet systems.

\section{Magnetics BACKGROUND}

This mathematical discussion uses the following conventions: Bold font denotes vectors (e.g., a, A) and blackboard font matrices (e.g., $\mathbb{B})$. The transpose is denoted $\mathbf{a}^{\top}$ or $\mathbb{A}^{\top}$. The vector 2-norm is $\|\mathbf{a}\|$, normalized direction (i.e., $\mathbf{a}\|\mathbf{a}\|^{-1}$ ) is $\hat{\mathbf{a}}$, inner product is $\mathbf{a} \cdot \mathbf{b}$, and cross product is $\mathbf{a} \times \mathbf{b}$, which is sometimes written in the skew-symmetric matrix form $\mathbb{S}(\mathbf{a}) \mathbf{b}$.

\section{A. Magnetic Dipole Equations}

The field produced by a magnetized object or a solenoid can be well modeled in regions outside of the object's bounding sphere (i.e., the smallest sphere that encapsulates the magnetic source) with a multipole field expansion [18]. The first term in this expansion, the dipole field, is

$$
\mathbf{B}_{i}=\frac{\mu_{0}}{4 \pi\left\|\mathbf{p}_{i, j}\right\|^{3}}\left(3 \hat{\mathbf{p}}_{i, j} \hat{\mathbf{p}}_{i, j}^{\top}-\mathbb{I}\right) \mathbf{m}_{j}=\frac{\mu_{0}}{4 \pi} \mathbb{P}_{i, j} \mathbf{m}_{j},
$$

where: $\mathbf{B}_{i}$ is the magnetic field at position $i$ that is produced by a dipole moment $\mathbf{m}_{j}$ at position $j ; \mathbf{p}_{i, j}$ is the displacement vector from position $j$ to position $i$ (i.e., the location of the calculated field $\mathbf{B}_{i}$ with respect to dipole $\mathbf{m}_{j}$ ); the $3 \times 3$ matrix $\mathbb{P}_{i, j}$ is a mapping function that is only dependent on the displacement $\mathbf{p}_{i, j}$, and $\mathbb{I}$ is the $3 \times 3$ identity matrix. Higher-order terms in the multipole expansion decay rapidly with distance, are neglected in this analysis, and would not fundamentally change the results.

Griffiths [18] provides relationships for torque and force. The torque $\mathcal{T}_{i}$ on a magnetic dipole $\mathbf{m}_{i}$ in a field $\mathbf{B}_{i}$ is

$$
\mathcal{T}_{i}=\mathbf{m}_{i} \times \mathbf{B}_{i}=\mathbb{S}\left(\mathbf{m}_{i}\right) \mathbf{B}_{i}
$$

In general, the force $\mathbf{F}_{i}$ on a magnetic dipole $\mathbf{m}_{i}$ is

$$
\mathbf{F}_{i}=\left(\mathbf{m}_{i} \cdot \nabla\right) \mathbf{B}_{i},
$$

where $\nabla$ is the vector gradient operator. If the magnetic field is produced by another dipole source $\mathbf{m}_{j}$, the force on dipole $\mathbf{m}_{i}$ from dipole $\mathbf{m}_{j}$ is

$$
\begin{aligned}
\mathbf{F}_{i}= & \frac{3 \mu_{0}}{4 \pi\left\|\mathbf{p}_{i, j}\right\|^{4}}\left(\mathbf{m}_{i} \hat{\mathbf{p}}_{i, j}^{\top}+\hat{\mathbf{p}}_{i, j} \mathbf{m}_{j}^{\top}\right. \\
& \left.+\left(\hat{\mathbf{p}}_{i, j} \cdot \mathbf{m}_{i}\right)\left(\mathbb{I}-5 \hat{\mathbf{p}}_{i, j} \hat{\mathbf{p}}_{i, j}^{\top}\right)\right) \mathbf{m}_{j} \\
= & \frac{3 \mu_{0}}{4 \pi} \mathbb{F}_{i, j} \mathbf{m}_{j} .
\end{aligned}
$$

The $3 \times 3$ matrix $\mathbb{F}_{i, j}$ is a mapping function that is dependent on both the displacement $\mathbf{p}_{i, j}$ and the dipole moment $\mathbf{m}_{i}$. When $\mathbf{p}_{i, j}$ is orthogonal to $\mathbf{m}_{i}, \mathbb{F}_{i, j}$ is singular and no force parallel to the $\mathbf{p}_{i, j} \times \mathbf{m}_{i}$ direction can be generated [17]

\section{B. The Dipole Moment of a Single Omnimagnet}

The dipole moment of an Omnimagnet, which is derived in [16], is composed of two main parts: the dipole moment of the solenoids and the dipole moment of the ferromagnetic core, which is magnetized by the solenoids. These two parts are both linear with current, assuming the core operates in its linear region, and can be written as

$$
\mathbf{m}_{o}=\mathbb{M}_{o} \mathbf{I}_{o}
$$

where $\mathbb{M}_{o}$ is a $3 \times 3$ matrix mapping the three applied currents, which are packed into the vector $\mathbf{I}_{o}$, to the resulting dipole moment $\mathbf{m}_{o} . \mathbb{M}_{o}$ is diagonal in the Omnimagnet's frame and can be easily inverted. Since the average magnetic field in a spherical volume is the magnetic-field's value at the sphere's center [18], if an Omnimagnet is placed in an external field, the perturbed dipole moment with the additional core magnetization is

$$
\mathbf{m}_{o}=\mathbb{M}_{o} \mathbf{I}_{o}+\frac{4 \pi}{\mu_{0}} R^{3} \mathbf{B}_{\mathrm{ext}}
$$


where $R$ is the radius of the Omnimagnet's core and $\mathbf{B}_{\text {ext }}$ is the external sources' magnetic field at the core's center [16]. The derivation of (6) assumes the core has negligible remanence and is unsaturated.

\section{Theory of Multiple-OMnimagnet Systems}

The combined effect of all of the field sources and the coupling between the Omnimagnet cores needs to be considered when using multiple Omnimagnets to control one or more magnetic tools. Let there be $N$ Omnimagnets, with dipole moments $\mathbf{m}_{o 1} \ldots \mathbf{m}_{o N}$ as determined by (6), and $L$ permanent-magnet tools, with dipole moments of $\mathbf{m}_{t 1} \ldots \mathbf{m}_{t L}$, which are located far enough from the Omnimagnets to be considered point-dipole sources [19]. The total external magnetic field at the center of the $i^{\text {th }}$ Omnimagnet's core is

$$
\mathbf{B}_{\mathrm{ext}, i}=\frac{\mu_{o}}{4 \pi}\left(\sum_{k=1}^{L} \mathbb{P}_{o i, t k} \mathbf{m}_{t k}+\sum_{\substack{j=1, j \neq i}}^{N} \mathbb{P}_{o i, o j} \mathbf{m}_{o j}\right),
$$

where $\mathbb{P}_{o i, o j}$ is the dipole displacement matrix from the $j^{\text {th }}$ to the $i^{\text {th }}$ Omnimagnet and $\mathbb{P}_{o i, t k}$ is the dipole displacement matrix from the $k^{\text {th }}$ permanent-magnet tool to the $i^{\text {th }}$ Omnimagnet. Combining (6) and (7), the coupled algebraic equations describing the dipole moment of the $i^{\text {th }}$ Omnimagnet $\mathbf{m}_{o i}$ are

$\mathbf{m}_{o i}=\mathbb{M}_{o i} \mathbf{I}_{o i}+R_{i}^{3}\left(\sum_{k=1}^{L} \mathbb{P}_{o i, t k} \mathbf{m}_{t k}+\sum_{\substack{j=1, j \neq i}}^{N} \mathbb{P}_{o i, o j} \mathbf{m}_{o j}\right)$.

Defining $\mathbf{m}$ as the vector packing of all of the Omnimagnets' dipole moments and $\mathbf{I}$ as the vector packing of all of the Omnimagnets' currents, these $3 N$ algebraic equations (8) can be extended to a matrix equation for the whole system

$$
\begin{aligned}
& \mathbf{m}=\left[\begin{array}{cccc}
\mathbb{O} & R_{1}^{3} \mathbb{P}_{o 1, o 2} & R_{1}^{3} \mathbb{P}_{o 1, o 3} & \\
R_{2}^{3} \mathbb{P}_{o 2, o 1} & \mathbb{O} & R_{2}^{3} \mathbb{P}_{o 2, o 3} & \cdots \\
R_{3}^{3} \mathbb{P}_{o 3, o 1} & R_{3}^{3} \mathbb{P}_{o 3, o 2} & \mathbb{O} & \\
& \vdots & & \ddots
\end{array}\right] \mathbf{m} \\
& +\left[\begin{array}{ccc}
R_{1}^{3} \mathbb{P}_{o 1, t 1} & \cdots & R_{1}^{3} \mathbb{P}_{o 1, t L} \\
\vdots & \ddots & \vdots \\
R_{N}^{3} \mathbb{P}_{o N, t 1} & \cdots & R_{N}^{3} \mathbb{P}_{o N, t L}
\end{array}\right]\left[\begin{array}{c}
\mathbf{m}_{t 1} \\
\vdots \\
\mathbf{m}_{t L}
\end{array}\right] \\
& +\left[\begin{array}{ccc}
\mathbb{M}_{\mathrm{o} 1} & & \mathbb{O} \\
& \ddots & \\
\mathbb{O} & & \mathbb{M}_{\mathrm{oN}}
\end{array}\right] \mathbf{I}
\end{aligned}
$$

where $\mathbb{O}$ denotes the appropriately sized zero padding. Thus, the dipole moments are given by the affine equation

$$
\mathbb{D} \mathbf{m}=\mathbb{M} \mathbf{I}+\mathbf{m}_{\mathrm{T}},
$$

where

$$
\mathbb{D}=\left[\begin{array}{cccc}
\mathbb{I} & -R_{1}^{3} \mathbb{P}_{o 1, o 2} & -R_{1}^{3} \mathbb{P}_{o 1, o 3} & \\
-R_{2}^{3} \mathbb{P}_{o 2, o 1} & \mathbb{I} & -R_{2}^{3} \mathbb{P}_{o 2, o 3} & \cdots \\
-R_{3}^{3} \mathbb{P}_{o 3, o 1} & -R_{3}^{3} \mathbb{P}_{o 3, o 2} & \mathbb{I} & \\
& \vdots & & \ddots
\end{array}\right]
$$

encodes the Omnimagnet coupling,

$$
\mathbb{M}=\left[\begin{array}{ccc}
\mathbb{M}_{o 1} & & \mathbb{O} \\
& \ddots & \\
\mathbb{O} & & \mathbb{M}_{o N}
\end{array}\right]
$$

describes the dipole moments of each Omnimagnet in the absence of magnetic coupling, and is block-diagonal, and

$$
\mathbf{m}_{\mathrm{T}}=\left[\begin{array}{ccc}
R_{1}^{3} \mathbb{P}_{o 1, t 1} & \cdots & R_{1}^{3} \mathbb{P}_{o 1, t L} \\
\vdots & \ddots & \vdots \\
R_{N}^{3} \mathbb{P}_{o N, t 1} & \cdots & R_{N}^{3} \mathbb{P}_{o N, t L}
\end{array}\right]\left[\begin{array}{c}
\mathbf{m}_{t 1} \\
\vdots \\
\mathbf{m}_{t L}
\end{array}\right]
$$

provides the Omnimagnet dipole moments due to the tools' field. For any physically realizable system, both $\mathbb{D}$ and $\mathbb{M}$ are full rank and invertible. When the magnitude of $\mathbf{m}_{\mathrm{T}}$ is much smaller than $\mathbb{M I}$, the tools' contribution can be neglected, and (10) becomes linear with current.

These equations assume the tools are permanent magnets. A soft-magnetic tool, not near saturation, will have a dipole moment that varies linearly with the applied field, and the reformulated system will be linear with current. Moreover, the perturbation to the Omnimagnet's dipole moment from the tool's field will be proportional to $V_{\mathrm{T}} \mathbb{P}^{2} \quad\left(V_{\mathrm{T}}\right.$ is the tool's volume), which decays as $\|\mathbf{p}\|^{-6}$, and will only be relevant for small separation distances. Thus, in many cases, the singular values of $V_{\mathrm{T}} \mathrm{P}^{2}$ will be much smaller than the singular values of $\mathbb{M}$, and the effect of the tools' field on the Omnimagnets can be neglected completely.

To determine the dipole moments resulting from a set of currents, $\mathbb{D}^{-1}$ must be computed, and needs to be updated if any of the Omnimagnets' relative positions change. To determine the currents to produce a set of dipole moments, $\mathrm{M}^{-1}$ must be computed, and must be updated if any of the the Omnimagnets' orientations change. Because of its structure, $\mathbb{M}^{-1}$ can be updated to reflect a change in an Omnimagnet's orientation more efficiently than $\mathbb{D}^{-1}$ can be updated to reflect a change in an Omnimagnet's position. This difference could become important for systems that incorporate a large number of Omnimagnets, since, as will be discussed next, the control choice can be calculated using either inverse.

Once the magnetic coupling is accounted for, superposition can be applied to the resulting set of dipole fields. Thus, the field applied by the Omnimagnets at a specific location (the location of the tool) is

$$
\mathbf{B}_{t}=\frac{\mu_{0}}{4 \pi}\left[\begin{array}{lll}
\mathbb{P}_{t, o 1} & \cdots & \mathbb{P}_{t, o N}
\end{array}\right] \mathbf{m} .
$$

Therefore, the torque on a single permanent-magnet tool is

$$
\mathcal{T}_{t}=\frac{\mu_{0}}{4 \pi} \mathbb{S}\left(\mathbf{m}_{t}\right)\left[\begin{array}{lll}
\mathbb{P}_{t, o 1} & \cdots & \mathbb{P}_{t, o N}
\end{array}\right] \mathbf{m}
$$


and the force on a single permanent-magnet tool is

$$
\mathbf{F}_{t}=\frac{3 \mu_{0}}{4 \pi}\left[\begin{array}{lll}
\mathbb{F}_{t, o 1} & \cdots & \mathbb{F}_{t, o N}
\end{array}\right] \mathbf{m} .
$$

To command a field (with which a magnetized object will attempt to align) and a force (e.g., [12], [15])

$$
\left[\begin{array}{l}
\mathbf{B}_{t} \\
\mathbf{F}_{t}
\end{array}\right]=\frac{\mu_{0}}{4 \pi}\left[\begin{array}{cc}
\mathbb{I} & \mathbb{O} \\
\mathbb{O} & 3 \mathbb{I}
\end{array}\right]\left[\begin{array}{lll}
\mathbb{P}_{t, 1} & \cdots & \mathbb{P}_{t, N} \\
\mathbb{F}_{t, 1} & \cdots & \mathbb{F}_{t, N}
\end{array}\right] \mathbf{m} .
$$

Alternately, Equations (15) and (16) can be stacked for the wrench on a single permanent-magnet tool

$$
\left[\begin{array}{l}
\mathcal{T}_{t} \\
\mathbf{F}_{t}
\end{array}\right]=\frac{\mu_{0}}{4 \pi}\left[\begin{array}{cc}
\mathbb{S}\left(\mathbf{m}_{t}\right) & \mathbb{O} \\
\mathbb{O} & 3 \mathbb{I}
\end{array}\right]\left[\begin{array}{lll}
\mathbb{P}_{t, o 1} & \cdots & \mathbb{P}_{t, o N} \\
\mathbb{F}_{t, o 1} & \cdots & \mathbb{F}_{t, o N}
\end{array}\right],
$$

but this wrench only constitutes 5-DOF due to the crossproduct in (2). Thus, it is not possible to apply a torque about the tool's dipole moment, leaving the the field in that direction unconstrained. However, 6-DOF control is possible if the tool contains multiple permanent magnets [14], [20]. To achieve 6-DOF control on a tool consisting of $L$ rigidly affixed permanent magnets, (15) and (16) combine as

$$
\begin{aligned}
& {\left[\begin{array}{l}
\mathcal{T}_{t} \\
\mathbf{F}_{t}
\end{array}\right]=\frac{\mu_{0}}{4 \pi}\left[\begin{array}{ccccc}
\mathbb{S}\left(\mathbf{m}_{t 1}\right) & \mathbb{S}\left(\mathbf{r}_{t 1}\right) & \ldots & \mathbb{S}\left(\mathbf{m}_{t L}\right) & \mathbb{S}\left(\mathbf{r}_{t L}\right) \\
\mathbb{O} & 3 \mathbb{I} & \ldots & \mathbb{O} & 3 \mathbb{I}
\end{array}\right]} \\
& {\left[\begin{array}{ccc} 
& & \\
\mathbb{P}_{t 1, o 1} & \cdots & \mathbb{P}_{t 1, o N} \\
\mathbb{F}_{t 1, o 1} & \cdots & \mathbb{F}_{t 1, o N} \\
\vdots & \vdots & \vdots \\
\mathbb{P}_{t L, o 1} & \cdots & \mathbb{P}_{t L, o N} \\
\mathbb{F}_{t L, o 1} & \cdots & \mathbb{F}_{t L, o N}
\end{array}\right] \mathbf{m},}
\end{aligned}
$$

where $\mathbf{r}_{t i}$ is the displacement vector from the tool's center of mass to the center of the $i^{\text {th }}$ affixed magnet.

Each of equations (14)-(19), and their various stacked and linear combinations, can be expressed in the form

$$
\mathbf{z}=\mathbb{A} \mathbf{m},
$$

where $\mathbf{z}$ is the output and $\mathbb{A}$ is the appropriate actuation matrix from above that maps the Omnimagnet dipole moments to the output. The output can be described as a function of the applied current by combining (10) and (20)

$$
\mathbf{z}=\mathbb{A D}^{-1}\left(\mathbb{M I}+\mathbf{m}_{\mathrm{T}}\right)=\mathbb{A D}^{-1} \mathbb{M I}+\mathbf{z}_{\mathrm{T}},
$$

where $\mathbf{z}_{\mathrm{T}}$ is due to the tools' field magnetizing the cores. In many cases, $\mathbf{z}_{\mathrm{T}}$ is negligible because it decays with $\|\mathbf{p}\|^{-6}$ for field or torque and decays with $\|\mathbf{p}\|^{-7}$ for force.

For some desired output $\mathbf{z}_{\mathrm{des}}$, the direct solution to (21) computes the minimum-2-norm solution for the dipole moment vector $\mathbf{m}$ and determines the currents to achieve it:

$$
\mathbf{I}=\mathbb{M}^{-1}\left(\mathbb{D A}^{\dagger} \mathbf{z}_{\mathrm{des}}-\mathbf{m}_{\mathrm{T}}\right)
$$

where the $\dagger$ symbolizes a Moore-Penrose generalized inverse, i.e., $\mathbb{A}^{\dagger}=\left(\mathbb{A}^{\top} \mathbb{A}\right)^{-1} \mathbb{A}^{\top}$. This solution is convenient because it does not require the explicit inverse of $\mathbb{D}$ (although it can be shown that its inverse always exists for physically realizable systems). If no solution to (22) exists (e.g., if the system is under-actuated), then the generalized inverse finds the set of currents $\mathbf{I}$ that minimizes the 2-norm of the difference between $\mathbf{z}$ and $\mathbf{z}_{\mathrm{des}}$.

For an over-actuated system, a minimum-dipole-moment solution is not the only option. Since current is the command input, seeking a solution that tends to minimize the control current, rather than dipole moments, could have thermal and power advantages. To minimize a quadratic function of current (i.e., $\mathbf{I}^{\top} \mathbb{W} \mathbf{I}$ ), we define a positive-definite symmetric weighting matrix $\mathbb{W}$ and use the change of variable $\mathbf{y}=$ $\mathbb{W}^{1 / 2} \mathbf{I}$. With this substitution, (21) becomes

$$
\mathbf{z}-\mathbf{z}_{\mathrm{T}}=\mathbb{A D}^{-1} \mathbb{M W}^{-1 / 2} \mathbf{y}
$$

and the solution for the control current vector I that minimizes the 2-norm of the weighted currents $\mathbf{y}$ and achieving a desired output $\mathbf{z}_{\text {des }}$ is

$$
\mathbf{I}=\mathbb{W}^{-1 / 2}\left(\mathbb{A D}^{-1} \mathbb{M W}^{-1 / 2}\right)^{\dagger}\left(\mathbf{z}_{\mathrm{des}}-\mathbf{z}_{\mathrm{T}}\right)
$$

Again, if no solution to (24) exists, then the generalized inverse finds the current vector $\mathbf{I}$ that minimizes the 2 norm of the difference between $\mathbf{z}$ and $\mathbf{z}_{\text {des }}$. The choice of $\mathbb{W}$ is arbitrary. Choosing a diagonal matrix packing of the solenoid resistances minimizes the electrical power required for a desired output. In a power-constrained system, this maximizes the capability.

\section{WORKSPACE ANALYSIS}

By considering the singular values of the actuation matrix for a field and force output, we are able to analyze the condition of the workspace. With two or more Omnimagnets it is possible to achieve 5-DOF manipulation of a single permanent-magnet tool at every position. However, there are some caveats to this statement. For example, if the tool and all of the Omnimagnets form a line, and if the dipole moment of the tool is orthogonal to this line, then it is not possible to apply a force out of the span of $\left\{\hat{\mathbf{p}}, \hat{\mathbf{m}}_{t}\right\}$, as is the case for manipulation with a single Omnimagnet [17]. Any pose that is close to this pose will be poorly conditioned. Furthermore, consider a system-bounding-sphere encompassing all of the Omnimagnets. A multipole expansion of the resulting field at locations outside of this system-bounding-sphere reveals the differences between the net field and a point-dipole field decays rapidly with the distance. Therefore, at large distances, the manipulability will quickly converge to that of a single dipole source, which has been quantified in [17] and is fundamentally only 3-DOF. Thus, the dexterous workspace of any collection of Omnimagnets is confined to locations within approximately 1.5 system-bounding-sphere radii and to configurations where the Omnimagnets and the tool are not collinear.

With two Omnimagnets the field-force actuation matrix $\mathbb{A}$ from (17) is square, and it is possible to achieve 5-DOF over a limited range of tool orientations as shown in Fig. 2. Unfortunately, at every location, there is a continuous set of tool orientations that are singular. Other systems with only six control inputs have reported similar orientation-dependent issues [15], [21]. However, this issue does not appear in the 


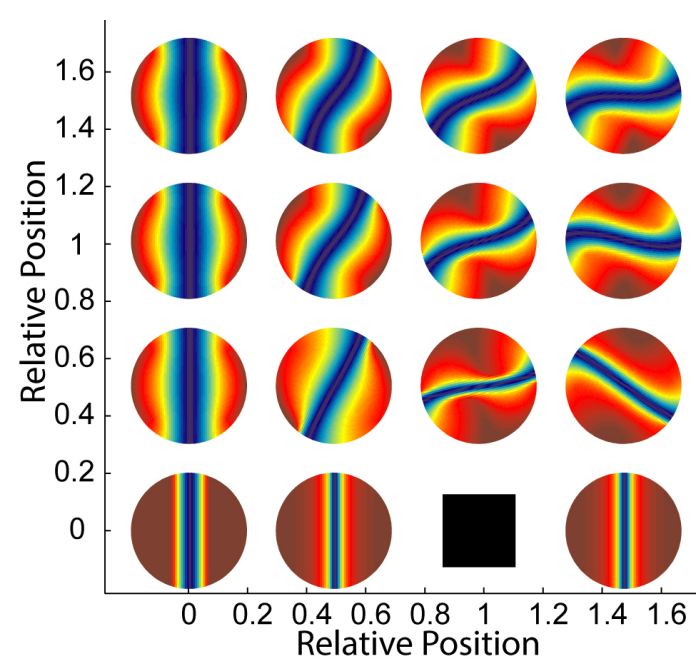

Fig. 2. The orientation-dependent minimum singular values for fifteen tool locations with two Omnimagnets at $(1,0)$ and $(-1,0)$ (the Omnimagnet at $(1,0)$ is represented by the black square). The other four quadrants are reflections of this quadrant. Each circle represents a single position at the center with a hemisphere of tool orientations, with the center corresponding to the tool's dipole moment pointing directly out of the plane and the edge corresponding to the tool's dipole moment lying in the plane. At each location, the blue line-like regions correspond to singular tool orientations and the red hemispherical regions correspond to fully manipulable orientations. With only two Omnimagnets there are many tool orientations where 5-DOF manipulation is possible, but there is always a small set of singular tool orientations where the manipulability is reduced to $4-\mathrm{DOF}$.

eight-coil systems developed in [12] and [13]. This suggests that the root cause of the singularities in the six-input systems is not a geometric issue, as was suggested by Meeker et al. [15], but, rather, is due to not having enough inputs to fully control the magnetic fields produced.

To investigate the root cause of these orientation singularities, we look at the systems from a magnetics perspective rather than from a mechanics perspective. To achieve a force on a permanent magnet that is independent of the field applied, it is necessary to have full control of both the field and the field Jacobian-commonly referred to as the field gradient. (We diverge from common usage because we wish to be clear that the field Jacobian is a $3 \times 3$ matrix, not a vector.) Maxwell's equations state that, $\boldsymbol{\nabla} \cdot \mathbf{B}=0$ everywhere and $\boldsymbol{\nabla} \times \mathbf{B}=\mathbf{0}$ in current-free space (i.e., where we will be manipulating the tool), and as a result, the field Jacobian will be trace-free and symmetric, respectively. With these constraints, a static (i.e., the coils are in a fixed geometry) magnetic-manipulation system requires 3-DOF to fully control the field and 5-DOF to fully control the field Jacobian. Therefore, every static system that has less than eight current inputs is not capable of fully controlling the 8-DOF magnetic-field and field-Jacobian system. Thus, the six-coil systems discussed above are actually under-actuated. The result is they have singular tool orientations when viewed from a force-torque or force-field perspective.

Indeed, numerical simulation shows that an actuation matrix with three non-collinear Omnimagnets (nine inputs) is never identically singular, but poorly conditioned orientations still exist for tool positions that are coplanar with the Omnimagnet positions. With four or more Omnimagnets, it is possible to arrange them such that the tool and the Omnimagnets can never be coplanar (e.g., place the Omnimagnets at the vertices of a triangular pyramid), and simulations show that the actuation matrix for this system is well conditioned in the maximum-inscribing-sphere (i.e., the largest sphere that can be placed inside the arrangement without containing any of the Omnimagnets) for all possible tool orientations. Clearly, adding additional Omnimagnets can only enhance the conditioning of the actuation matrix.

\section{EXPERIMENTAL DEMONSTRATION}

To demonstrate the reconfigurability of the system, we chose four different configurations of Omnimagnets as shown in Fig. 3. We begin our experiments with three Omnimagnets, then add a fourth, and then a fifth. Finally, we reconfigure the third arrangement so the two stacked magnets are beyond the far end of the tank; the rectangular shape of the tank makes this more than a trivial rotation. For each of these configurations, the Omnimagnets were arranged by hand to accommodate the geometry of the water tank and the stereo-vision cameras, their positions were then measured with a ruler, and the system configuration was updated in the control software. No precise placement, measurement, or characterization was performed.

Two different styles of Omnimagnet were used, and they work seamlessly together. We use one large Omnimagnet, and four smaller Omnimagnets. The large Omnimagnet is the same device used in [16] and [17]; it has a cubic dimension of $176 \mathrm{~mm}$, a core radius of $50.8 \mathrm{~mm}$, and a maximum dipole strength of $200 \mathrm{~A} \cdot \mathrm{m}^{2}$ at $8 \mathrm{~A}$. The smaller Omnimagnets have a cubic dimension of $127 \mathrm{~mm}$, a core radius of $38 \mathrm{~mm}$, and a maximum dipole strength of $50 \mathrm{~A} \cdot \mathrm{m}^{2}$ at $8 \mathrm{~A}$.

In each configuration, the Omnimagnets are commanded to drive a semiboyant capsule around a cubic trajectory, while maintaining a vertical orientation, then commanded to hold the capsule in one position while rotating it completely around the world $x$ - and $y$-axis, sequentially. The geometry of the capsule is provided in [22]. It has a dipole moment of $0.126 \mathrm{~A} \cdot \mathrm{m}^{2}$, and a dry weight of $0.0153 \mathrm{~N}$, which is reduced to an apparent weight of $0.0005 \mathrm{~N}$ when submerged in water. The capsule is painted green and tracked at $40 \mathrm{~Hz}$ with a custom color tracking stereo-vision system, comprised of two orthogonal Point Grey FL3-U3-13S2C-CS cameras, with a reproduction error of $0.5 \mathrm{~mm}$. The frame rate and absolute accuracy (on the order of a few millimeters) are limited by the color detection and segmentation method, and could be improved by using a dynamic region-of-interest, but are adequate for these experiments.

The position error relative to the desired trajectory is converted to a desired force by a PID controller. This force along with the desired heading are converted to the required currents using the method of (17) with (24), and the field magnitude $\|\mathbf{B}\|$ was chosen to be $1.5 \mathrm{mT}$, which was empirically determined. The required currents are converted to an analog signal with an Advantech D-A card (PCI-1724U), and 

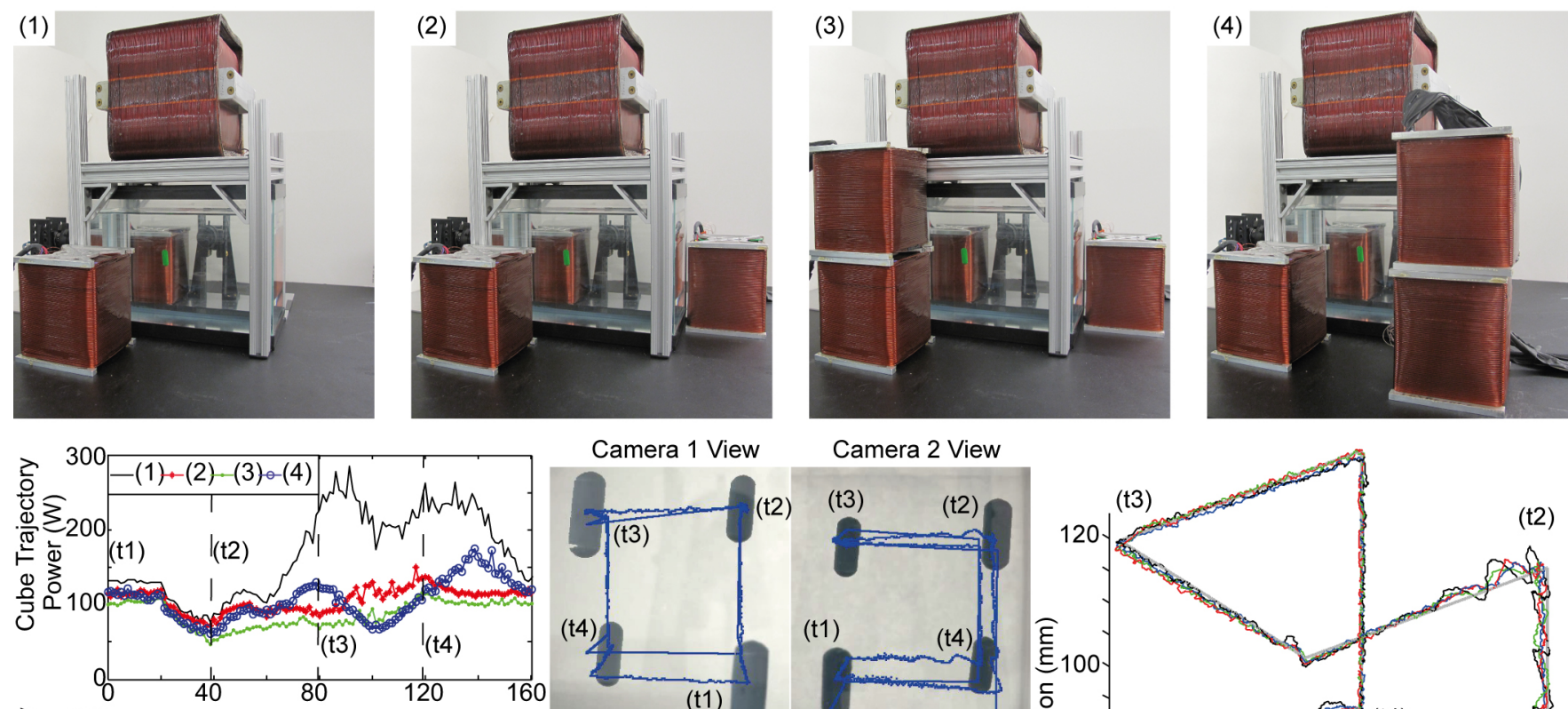

Camera 1 View

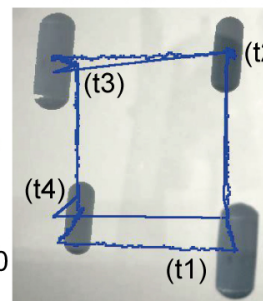

Camera 2 View

(t2)

(t3)
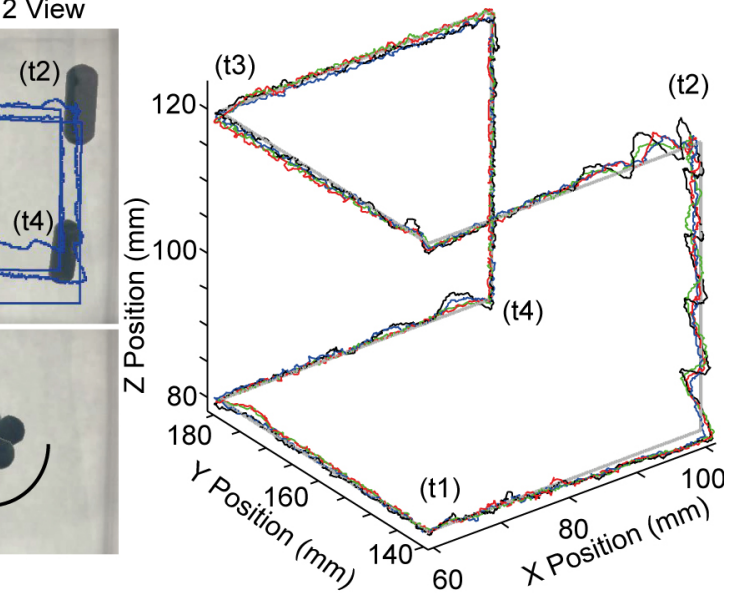

Fig. 3. (top) The four different Omnimagnet configurations tested. (bottom left) The power required to execute the trajectories. (bottom center) A time overlay of the trajectories from the camera point of view. (bottom right) The tracked positions for each cubic trajectory overlaid on the commanded path.

the current control is accomplished with Advanced Motion Control current drives (AMC30A8).

In the experiments, we observe that the total system power required to perform the same task decreases with each additional Omnimagnet. The average power to complete the rectangular trajectory with three Omnimagnets is $168 \mathrm{~W}$ and drops to $107 \mathrm{~W}$ and $87 \mathrm{~W}$ as additional Omnimagnets are added (see setup 1, 2, and 3 in Fig. 3). Similarly, the rotational trajectory requires $183 \mathrm{~W}, 121 \mathrm{~W}$, and $109 \mathrm{~W}$ on average for three, four, and five Omnimagnets, respectively. Interestingly, the modification of setup 3 into setup 4 moved the two stacked Omnimagnets farther from the trajectory, increasing the average power required to $107 \mathrm{~W}$ for the rectangular and $130 \mathrm{~W}$ for the rotational trajectories. Videos of these trajectories are available at the author's website [23].

Since the positions and orientations of the Omnimagnets were not precisely measured, the individual strengths of the Omnimagnets have not been calibrated to account for manufacturing differences, and the field contributed by each Omnimagnet has small deviations from the dipole model, it was necessary to add the integration to eliminate the steady-state position error. Despite accumulating modeling errors, the method proposed is robust enough to stabilize the inherently unstable magnetic control system even while operating at the relatively slow update rate of $40 \mathrm{~Hz}$.

\section{CONCLUSION AND Discussion}

This paper makes three key contributions: First, it derives an analytical model for the magnetic coupling of ferromagnetic spheres. This is a fundamental contribution to magnetic analysis and can be used to predict the behaviors of magnetic colloids or other systems comprised of interacting magnetic spheres. Second, it shows that any system of static electromagnets must have at least eight inputs to exhibit full control of a magnetic tool in all locations and orientations. The shift from analyzing the system from a mechanical to a magnetic perspective will help future magnetic manipulation system designers analyze their systems more effectively. Third, it presents the first modular and reconfigurable magnetic manipulation system.

The applications for a modular and reconfigurable magnetic manipulation system are numerous. For example, current magnetically controlled catheter ablation procedures use $\mathrm{C}$-arm fluoroscopy to image the catheter for the physician, and current manipulation systems significantly limit the viewing angles of the $\mathrm{C}$-arm. One could imagine a multipleOmnimagnet system adjusting the positions of the electromagnets to provide the best perspectives to the physician. Continuing along the lines of ablation procedures, a typical procedure has two parts: navigation and ablation. During navigation, it is beneficial to have excellent control of the field in order to steer the catheter tip though the vasculature. 
During ablation, it is beneficial to have excellent control of the force in order to press the catheter tip into the ventricle wall ensuring good thermal connection. This system can maintain control of the catheter tip while relocating the Omnimagnets to optimize the actuation matrix for each task, e.g., making a strong field during navigation and strong gradients during ablation.

The system presented in this paper is modular, reconfigurable, and has an analytically described field and gradient. It is the most versatile magnetic-manipulation system to date. It can be easily adapted to new tasks and procedures, assembled in any configuration desired, and modified during use, with no time-intensive in situ empirical or numerical field characterization required.

Although we have not fully explored the topic, it seems possible that this versatility could come at a cost in terms of performance for a specific application. The omnidirectional nature of the Omnimagnets can be interpreted as them both being equally good at generating a magnetic field in any direction or being equally poor at generating a magnetic field in any direction. An electromagnet of comparable size that is designed to generate a field in only a single direction will certainly be stronger in that direction. It may be that for a specific single application, a custom electromagnet system will outperform the modular approach. However, the benefits of being able to generate multiple orthogonal and collocated dipole fields using Omnimagnets creates new possibilities in terms of dexterous magnetic manipulation that are still unexplored, and that could potentially outweigh the drawbacks.

\section{REFERENCES}

[1] E. H. Frei, "Medical applications of magnetism," Crit Rev Solid State Mat Sci, vol. 1, no. 3, pp. 381-407, Sep 1970.

[2] G. T. Gillies, R. C. Ritter, W. C. Broaddus, M. S. Grady, M. A. Howard III, and R. G. McNeil, "Magnetic manipulation instrumentation for medical physics research," Rev Sci Instrum, vol. 65, no. 3, pp. 533-562, Mar 1994

[3] B. J. Nelson, I. K. Kaliakatsos, and J. J. Abbott, "Microrobots for minimally invasive medicine," Апnи Rev Biomed Eng, vol. 12, no. 1, pp. 55-85, 2010.

[4] A. W. Jenkins and H. M. Parker, "Electromagnetic support arrangement with three-dimensional control. I. theoretical," J App Phys, vol. 30, no. 4, pp. S238-S239, Apr 1959.

[5] K. B. Yesin, K. Vollmers, and B. J. Nelson, "Modeling and control of untethered biomicrorobots in a fluidic environment using electromagnetic fields," Int J Rob Res, vol. 25, no. 5-6, pp. 527-536, May 2006.
[6] T. Honda, K. I. Arai, and K. Ishiyama, "Micro swimming mechanisms propelled by external magnetic fields," IEEE Trans Magn, vol. 32, no. 5, pp. 5085-5087, Sep 1996.

[7] S. M. Jeon, G. H. Jang, H. C. Choi, S. H. Park, and J. O. Park, "Utilization of magnetic gradients in a magnetic navigation system for the translational motion of a micro-robot in human blood vessels," IEEE Trans Magn, vol. 47, no. 10, pp. 2403-2406, Oct. 2011.

[8] T. P. L. Roberts, W. V. Hassenzahl, S. W. Hetts, and R. L. Arenson, "Remote control of catheter tip deflection: an opportunity for interventional MRI," Magn Reson Med, vol. 48, no. 6, pp. 1091-1095, 2002.

[9] J.-B. Mathieu, S. Martel, L. Yahia, G. Soulez, and G. Beaudoin, "Preliminary investigation of the feasibility of magnetic propulsion for future microdevices in blood vessels," Biomed Mater Eng, vol. 15, no. 5, pp. 367-374, Sep 2005.

[10] E. E. Covert, "Magnetic suspension and balance systems," IEEE Aero Elec Sys Mag, vol. 3, no. 5, pp. 14-22, May 1988.

[11] T. Nakamura and M. B. Khamesee, "A prototype mechanism for threedimensional levitated movement of a small magnet," IEEE ASME Trans Mechatron, vol. 2, no. 1, pp. 41-50, Mar 1997.

[12] M. P. Kummer, J. J. Abbott, B. E. Kratochvil, R. Borer, A. Sengul, and B. J. Nelson, "OctoMag: An electromagnetic system for 5-DOF wireless micromanipulation," IEEE Trans Robot, vol. 26, no. 6, pp. 1006-1017, Dec 2010.

[13] Y. Shachar, "Apparatus and method for shaped magnetic field control for catheter, guidance, control, and imaging," US Patent 8,027,714 B2, 2011.

[14] P. Berkelman and M. Dzadovsky, "Magnetic levitation over large translation and rotation ranges in all directions," IEEE/ASME Trans Mechatron, vol. 18, no. 1, pp. 44-52, Feb 2013.

[15] D. C. Meeker, E. H. Maslen, R. C. Ritter, and F. M. Creighton, "Optimal realization of arbitrary forces in a magnetic stereotaxis system," IEEE Trans Magn, vol. 32, no. 2, pp. 320-328, Mar. 1996.

[16] A. J. Petruska and J. J. Abbott, "Omnimagnet: An omnidirectional electromagnet for controlled dipole-field generation," IEEE Trans Magn, vol. 50, no. 7, pp. 1-10, Jul 2014.

[17] A. J. Petruska, A. W. Mahoney, and J. J. Abbott, "Remote manipulation with a stationary computer-controlled magnetic dipole source," IEEE Tran Robot, vol. 30, no. 5, pp. 1-6, Dec 2014.

[18] D. J. Griffiths, Introduction to Electrodynamics. Prentice Hall, 1999.

[19] A. J. Petruska and J. J. Abbott, "Optimal permanent-magnet geometries for dipole field approximation," IEEE Trans Magn, vol. 49, no. 2, pp. 811-819, Feb 2013.

[20] E. Diller, J. Giltinan, G. Z. Lum, Z. Ye, and M. Sitti, "Six-degrees-offreedom remote actuation of magnetic microrobots," in Proceedings of Robotics: Science and Systems, Berkeley, USA, July 2014.

[21] S. Afshar, M. B. Khamesee, and A. Khajepour, "Optimal configuration for electromagnets and coils in magnetic actuators," IEEE Trans Magn, vol. 49, no. 4, pp. 1372-1381, Apr 2013.

[22] A. W. Mahoney and J. J. Abbott, "5-dof manipulation of an untethered magnetic device in fluid using a single permanent magnet," in Proceedings of Robotics: Science and Systems, Berkeley, USA, July 2014.

[23] [Online]. Available: www.telerobotics.utah.edu/index.php/Research/ Omnimagnets 